\title{
USE OF HIGH ALTITUDE PLATFORM SYSTEMS TO AUGMENT GROUND BASED APNT SYSTEMS
}

\author{
Omar García-Crespillo*, Elisabeth Nossek*, Andreas Winterstein*, \\ Boubeker Belabbas* and Michael Meurer ${ }^{* \dagger}$ \\ ${ }^{*}$ Institute of Communication and Navigation, German Aerospace Center (DLR), Oberpfaffenhofen, Germany \\ ${ }^{\dagger}$ Chair of Navigation, RWTH Aachen University, Germany
}

\begin{abstract}
An Alternative Position Navigation and Timing (APNT) service is needed to backup satellite navigation for civil aviation in case of a radio frequency interference. Current APNT services rely on legacy systems and range sources based on ground stations and therefore, present limitations in terms of geometric diversity due to poor visibility. Moreover, there is still no straight forward solution how to maintain the correct synchronization of the ranging sources in case of GNSS outage. In this work, we propose the potential use of High Altitude Platform Systems (HAPS) first as pseudolites to enhance a ground APNT system. We evaluate the coverage of the platform and the enhancement in terms of dilution of precision. This lead to a ground-stratospheric APNT system with increased navigation service area. Besides, we propose these platforms for time synchronization and for integrity monitoring of the ground stations.
\end{abstract}

\section{Introduction}

The provision of a robust Position, Navigation and Timing (PNT) service is crucial for the Air Traffic Management (ATM), and plays an essential role especially in civil aviation, where safety is a major concern. In this sense, the use of Global Navigation Satellite Systems (GNSS) has been identified by SESAR [1] and NextGen [2] as the primary future navigation system for civil aviation. However, due to the low power level of the GNSS signals, they can be easily jammed, causing an interruption in the navigation service. In fact, the high threat of having a Radio Frequency Interference (RFI) (i.e., jamming) has led to the need of a GNSS back-up system that aims at providing a navigation service for civil aviation in case that satellite navigation is not available: an Alternative Position, Navigation and

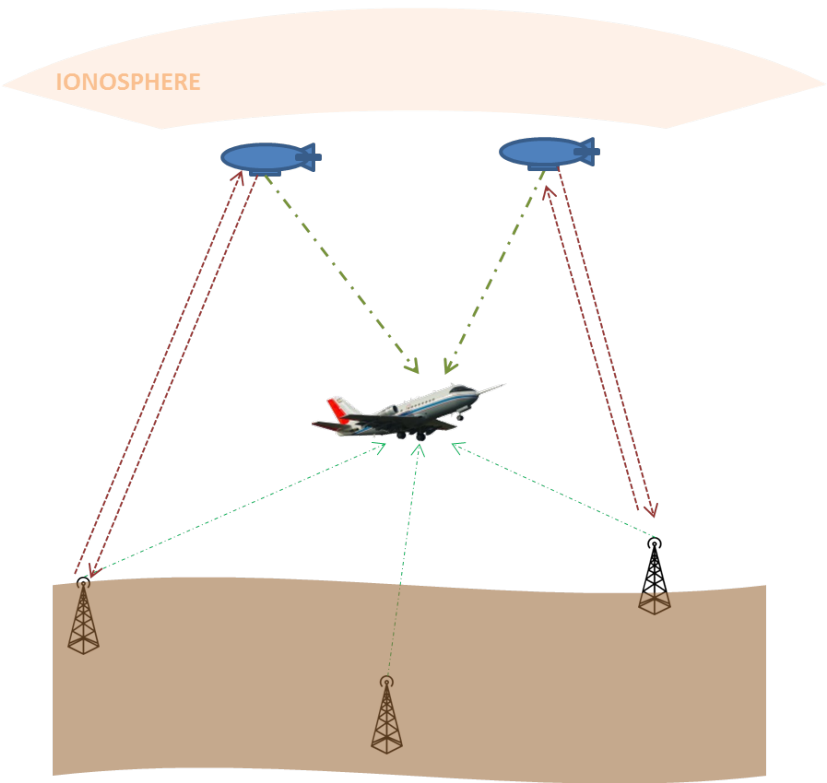

Figure 1. Ground-Stratospheric APNT System

Timing (APNT) system [3]. Currently, different approaches are under investigation that make use mainly of legacy radionavigation systems or other types of ground based ranging sources such as Distance Measuring Equipment (DME) or enhanced DME (eDME) [4], [5], Automatic Dependent Surveillance - Broadcast (ADS-B) [6], L-band Digital Aviation Communication System (LDACS) [7], and Universal Access Transceivers (UAT) [5], [8]. They are considered suitable options because their frequency band is different from the GNSS signals but they still belong to the radionavigation reserved spectrum. However, the visibility of ground stations is not homogeneous everywhere, especially at lower altitudes, and therefore, the required navigation performance may not be fulfilled due to the poor geometrical diversity. Moreover, there is still no established solution on how to provide the time synchronization of the ground 
stations in case of a longer GNSS outage.

In this sense, we consider an additional stratospheric system, called High Altitude Platform Systems (HAPS), that could work in combination with an existent ground APNT system. High Altitude Platform Systems (HAPS) are quasi-stationary platforms that operate from the stratosphere. In particular, they are expected to fly between 17 and $22 \mathrm{~km}$ high [9]. The HAPS have many of the advantages of a satellite, like direct line-of-sight visibility with a big portion of the earth surface, but they can operate at a lower cost and its maintenance or replacement can be easier performed. Because of that, many emerging applications have been proposed using HAPS, mainly related to wireless communication [10], but also for remote sensing, surveillance [11] and navigation [9]. In particular, in the field of navigation, HAPS have been proposed as a local augmentation system to support Global Navigation Satellite Systems (GNSS) [12], [13] and as a stratospheric GNSS-like pseudolite (stratolite) [14]. Also, [15] analysed the potential use of HAPS as an ADS-B receiver and for multilateration. Therefore, there are many different payloads that a HAPS can carry and we can think of a HAPS as a multiservice platform. However, as the main energy source for a long-term mission will come from the sun, the power constraint has to be taken into account. Moreover, the performance of the control system responsible of maintaining a static position against the stratospheric winds may limit the overall application performance, especially for navigation or positioning purposes.

In this paper, we consider the use of High Altitude Platform Systems (HAPS) to enhance and overcome some of the current limitations of APNT. First, due to the beneficial position of this platforms in the stratosphere, HAPS can guarantee line-ofsight visibility in a large coverage area to provide a pseudolite service in the air space. We analyse the impact in terms of dilution of precision when using several platforms in combination with the DME ground stations in Europe and assess the user position error sensibility to the HAPS position error. Finally, we propose the further use of HAPS as time synchronization provider and for integrity monitoring.

\section{Ground-Stratospheric APNT System}

The overview of the ground-stratospheric system proposed in this paper can be seen in Figure 1, where we can consider two different segments: Ground segment and stratospheric segment.

- Ground segment: The ground segment consist of different stations that are able to transmit a signal that is used as a ranging source by the aircraft. They could be from different sources and we assume that they transmit with respect to a common system time.

- Stratospheric segment: This segment includes one or several High Altitude Platform Systems (HAPS) that transmit ranging signals to the aerial vehicle. Additionally, the HAPS will include in the signal a navigation message with information about the exact position of the HAPS in a similar way as the ephemeris of satellites in GNSS. This position in the stratosphere (17-22 km) has many benefits for positioning: more robustness against interference and jamming, line-of-sight visibility within a large coverage area, lower cost in deployment and maintenance compared to satellites and signals free of ionospheric and multipath errors. Global Navigation Satellite System (GNSS) is used for the accurate positioning of the high altitude platform. We can consider that in the presence of a Radio Frequency Interference (RFI) that is affecting the GNSS reception at the airborne side, due to the longer distance and the pointing direction of the GNSS antenna on the HAPS, it would not be affected.

\section{HAPS Coverage Analysis}

In the following, we describe the geometric scenario for the HAPS and we analyze the corresponding link budget. From that, an example for the requirements of the antenna system and the necessary transmit power is obtained. Finally, the applicability of some simple antenna designs for the scenario is discussed.

\section{Geometric Scenario}

The study of the coverage of the HAPS can be done similar to the general approach used for satellite applications and is illustrated schematically in Figure 2. In our scenario, the transmitting HAPS is considered to be at height $h_{\mathrm{Tx}}$ above ground, the 


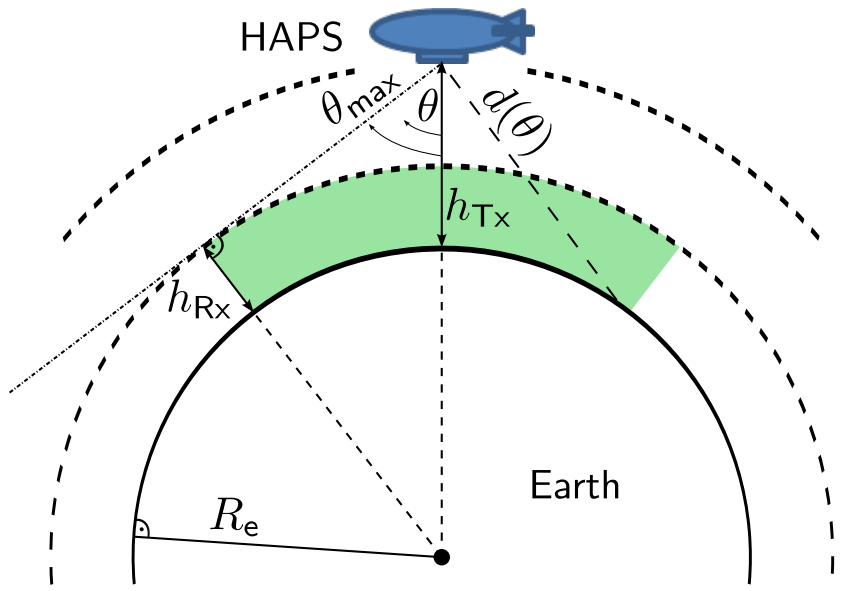

Figure 2. Geometric Coverage of the HAPS

maximum altitude of the receiving terminal where service shall be guaranteed is $h_{\mathrm{Rx}}$ and $R_{e}$ is the Earth's radius. Using this setup and considering the limiting case that the signal is received at $0^{\circ}$, we can calculate by trigonometry the maximum nadir angle to be covered as:

$$
\theta_{\max }=\sin ^{-1}\left(\frac{R_{e}+h_{\mathrm{Rx}}}{R_{e}+h_{\mathrm{Tx}}}\right) .
$$

Note that since $R_{e}>>h_{\mathrm{Tx}}>h_{\mathrm{Rx}}$, the angle $\theta_{\max }$ can get very close to $90^{\circ}$ as it can be seen in Table I for a HAPS height of $17 \mathrm{~km}$.

Within the coverage region, the HAPS always appears at elevation angles $\theta \geq 0^{\circ}$ as seen from the terminal. The maximum signal path length in this case occurs at the Earth's surface and is given by

$$
d_{\max }=\sqrt{2 R_{e}\left(h_{\mathrm{Tx}}-h_{\mathrm{Rx}}\right)+h_{\mathrm{Tx}}^{2}},
$$

whereas the path length for the maximum angle is shorter, reading

$$
d\left(\theta_{\max }\right)=\sqrt{2 R_{e}\left(h_{\mathrm{Tx}}-h_{\mathrm{Rx}}\right)+h_{\mathrm{Tx}}^{2}-h_{\mathrm{Rx}}^{2}} .
$$

The service area with respect to Earth's surface is computed as follows:

$$
S=2 \pi R_{e}^{2}\left(1-\cos \left(\frac{R_{e}+h_{\mathrm{Rx}}}{R_{e}+h_{\mathrm{Tx}}}\right)\right),
$$

Table I. Maximum Coverage Angle and Distance

\begin{tabular}{llll}
\hline Receiver Altitude & $5.000 \mathrm{ft}$ & $20.000 \mathrm{ft}$ & $30.000 \mathrm{ft}$ \\
\hline$\theta_{\max } /{ }^{\circ}$ & 86.01 & 86.65 & 87.16 \\
$d_{\max } / \mathrm{km}$ & 443.94 & 372.64 & 316.30 \\
\hline
\end{tabular}

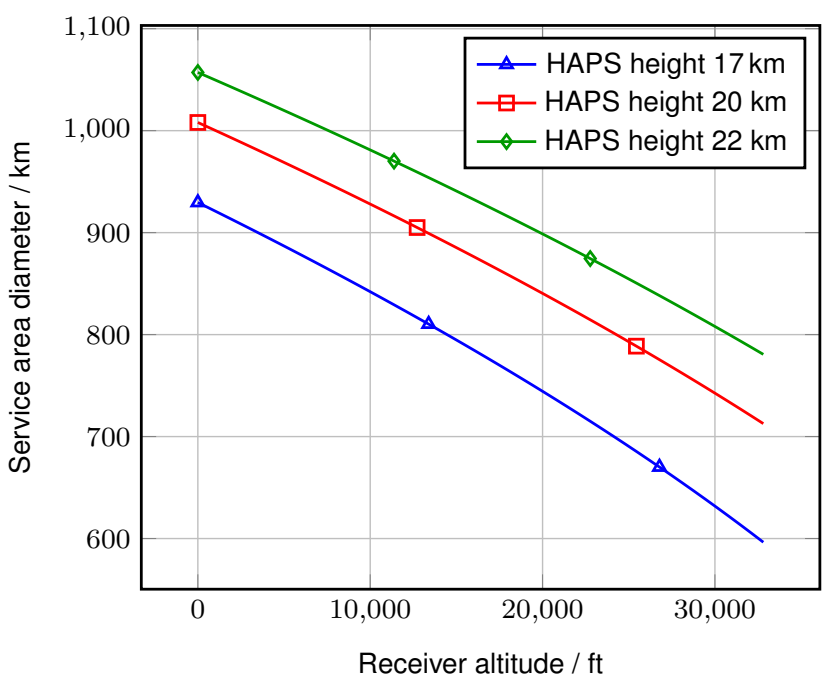

Figure 3. HAPS Service Area Diameter

and the diameter of this service area is found by:

$$
D=2 R_{e} \cos ^{-1}\left(\frac{R_{e}+h_{\mathrm{Rx}}}{R_{e}+h_{\mathrm{Tx}}}\right) .
$$

Figure 3 shows the different diameters of service areas for different HAPS heights and receiver altitudes.

\section{Link Budget Calculation}

The link budget between the HAPS and a terminal gives us the received power according to

$$
P_{\mathrm{Rx}}=\frac{P_{\mathrm{T} x} g_{\mathrm{T} \times} g_{\mathrm{Rx}}}{L_{\mathrm{Rx}} L_{\mathrm{T} \times} L_{\mathrm{FS}} L_{\mathrm{M}}} .
$$

It should be noted that linear power values have to be used in this equation, not the ones in $\mathrm{dB}$. This also applies to the following formulas in this section. Hereby, $P_{\mathrm{Tx}}$ is the total transmit power. $g_{\mathrm{Rx}}$ and $g_{\mathrm{Tx}}$ are the gain values of the receiver and transmitter antennas. $L_{\mathrm{Rx}}$ and $L_{\mathrm{Tx}}$ are the losses due to the receiver and transmitter systems (e.g. cable losses, impedance mismatch, etc.). $L_{\mathrm{M}}$ is a safety margin for unpredictable losses like atmospheric conditions, rain, scattering, etc. Finally, $L_{\mathrm{FS}}$ is the free-space path loss which is defined as

$$
L_{\mathrm{FS}}(\theta)=\left(\frac{4 \pi}{\lambda} d(\theta)\right)^{2},
$$

using the wavelength $\lambda$ of the signal frequency.

Since we could use different APNT signals for the HAPS, we will now make the following general assumptions as an example regarding the link budget calculation: The necessary received power shall be 
$P_{\mathrm{Rx}}=-100 \mathrm{dBm}$. System losses are assumed to be $L_{\mathrm{Rx}}=L_{\mathrm{Tx}}=3 \mathrm{~dB} . L_{\mathrm{M}}=7 \mathrm{~dB}$ is usually considered sufficient. Then, since there could be different types of receiving antennas, we assume a typical gain of $g_{\mathrm{Rx}}=-5 \mathrm{~dB}$. And we take a signal frequency of $1.164 \mathrm{GHz}$. The remaining variables are the transmit antenna gain $g_{\mathrm{T}_{\mathrm{X}}}(\theta)$ and the power $P_{\mathrm{T}_{\mathrm{X}}}$. Increasing transmit power is expensive and may cause interference with other systems. Thus, the design focus clearly lies on shaping the antenna pattern i.e. how $P_{\mathrm{Tx}}$ will be distributed.

\section{Antenna Pattern Considerations}

First, we want to find the shape of the desired antenna gain pattern $g_{\mathrm{Tx}}(\theta)$. We assume a loss-less antenna, i.e. all transmit power is radiated. Reformulating (6), we get

$$
g_{\mathrm{T} \times}(\theta)=\frac{P_{\mathrm{Rx}} L_{\mathrm{Rx}} L_{\mathrm{T} \times} L_{\mathrm{M}}}{g_{\mathrm{Rx}} P_{\mathrm{T} \times}} L_{\mathrm{FS}}(\theta),
$$

which shows that the ideal pattern should be proportional to the path loss. From antenna theory [16], we know that gain is normalized such that a closed surface integral over it evaluates to 1 . This makes the patterns of different antennas comparable.

In Figure 4, we can see an example of the ideal antenna pattern obtained for a coverage altitude design of $5.000 \mathrm{ft}$. Note that the angle $\theta$ is referred to the vertical axis, and it has rotational symmetry. Its gain increases to the maximum of $18.45 \mathrm{dBi}$ at $85.81^{\circ}$. Then, the gain drops shortly until the maximum angle $86.01^{\circ}$ and beyond that, it falls rapidly.

For comparison, Figure 4 depicts also the directive gain patterns of a half-isotropic radiator and a cosine shaped pattern. The former exists only in theory, the latter approximates the characteristic of a patch antenna looking towards $0^{\circ}$. The last pattern is that of a monopole over an infinite metallic ground plane [16]. Table II shows the transmit powers necessary for the different patterns to provide service to the high values of $\theta$. It can be seen that the necessary transmit power increases considerably for non-ideal patterns. Because the cosine-shaped pattern concentrates energy at the center, it shows the worst performance. The monopole achieves good performance for high steering angles. Its ground plane acts as a reflector and confines the field in the hemisphere below the HAPS. For a real, i.e. finite, plane there will be a certain spillover. However, the monopole

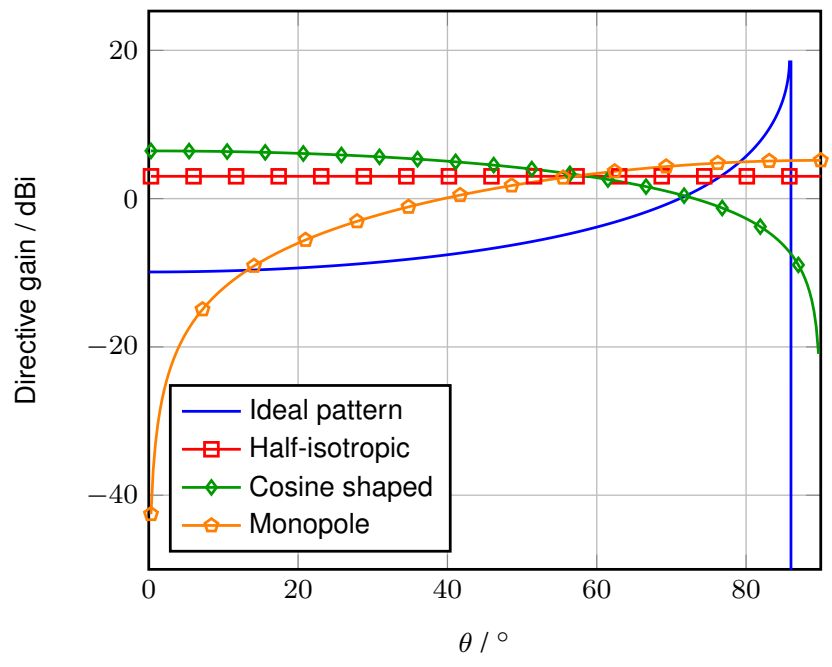

Figure 4. Comparison of Antenna Patterns

cannot provide service directly below the HAPS for $0^{\circ} \leq \theta<3^{\circ}$.

The ideal pattern is not realizable because of the abrupt slope. Concentrating most of the power close to $90^{\circ}$ and having such a broad pattern at the same time is a challenging design task. It is difficult to realize the desired coverage with a simple antenna design, as can be seen in Figure 4. In satellite communications, isoflux antennas are used to equalize path differences when illuminating earth. There are different implementation possibilities as shown in [17], [18], and [19]. However, these solutions are not applicable in our HAPS scenario for two reasons: First, the necessary ranges of $\theta$ in satellite communications are narrower, i.e. $\pm 15^{\circ}$ for GEO or $\pm 60^{\circ}$ for LEO satellites. Second, the relative differences in path length are much smaller. The gain difference between boresight and maximum is only up to $10 \mathrm{~dB}$ for a LEO [17] as opposed to the $28 \mathrm{~dB}$ in our case. It follows therefore that the HAPS application would require a specific antenna design. The development of such a specialized device is beyond the scope of this work.

Table II. Necessary Transmit Power

\begin{tabular}{lll}
\hline Pattern shape & $P_{\mathrm{Tx}} / \mathrm{dBm}$ & $P_{\mathrm{Rx}}\left(\theta=0^{\circ}\right) / \mathrm{dBm}$ \\
\hline Ideal & 46.26 & -100.00 \\
Half-isotropic & 61.71 & -71.66 \\
Cosine & 72.16 & -57.78 \\
Monopole & 59.59 & $-\infty$ \\
\hline
\end{tabular}


A possible approach would be to combine a monopole with a patch antenna to eliminate the area without service below the HAPS. Also conformal antenna structures may be considered as their patterns can be tailored to provide better coverage over the whole range of $\theta$.

\section{Dilution Of Precision Enhancement}

In this section, we analyze one of the major advantages of using HAPS as a ranging source for positioning. Due to the limited number of ground stations that are available for APNT, the resolution is not always enough to achieve a Dilution Of Precision (DOP) value that satisfies the performance requirements (e.g., RNP 0.3). We make two comparisons: first we consider the territory of Europe and the achieved HDOP values for a flying altitude of 20.000 $\mathrm{ft}$. This corresponds to the middle climbing/descent phase of commercial aircraft or to the flying altitude of some other smaller commercial or non-commercial aircrafts; second, we show the improvement for an initial climbing or a final descent approach phase near an airport at lower altitudes.

\section{HDOP over Europe}

The number of visible DME stations at an altitude of $20.000 \mathrm{ft}$ can be seen in Figure 5 for Europe. Although there are some parts, mainly in the center of Europe, where there is a good visibility of ground stations, there are other areas where the number of

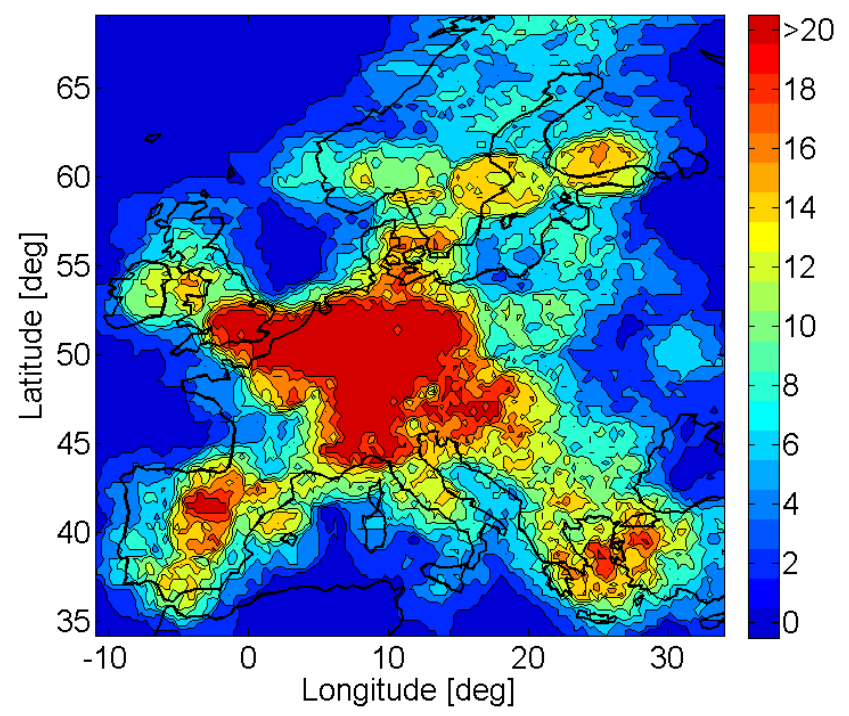

Figure 5. Visible DME Stations at 20.000 ft.

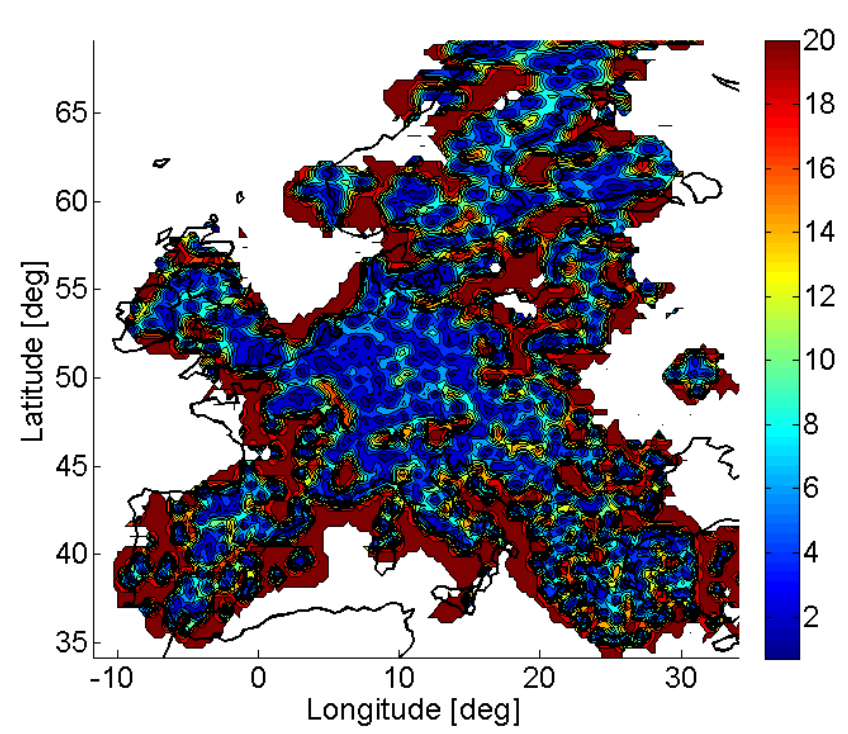

Figure 6. HDOP at $20.000 \mathrm{ft}$. with DME Stations

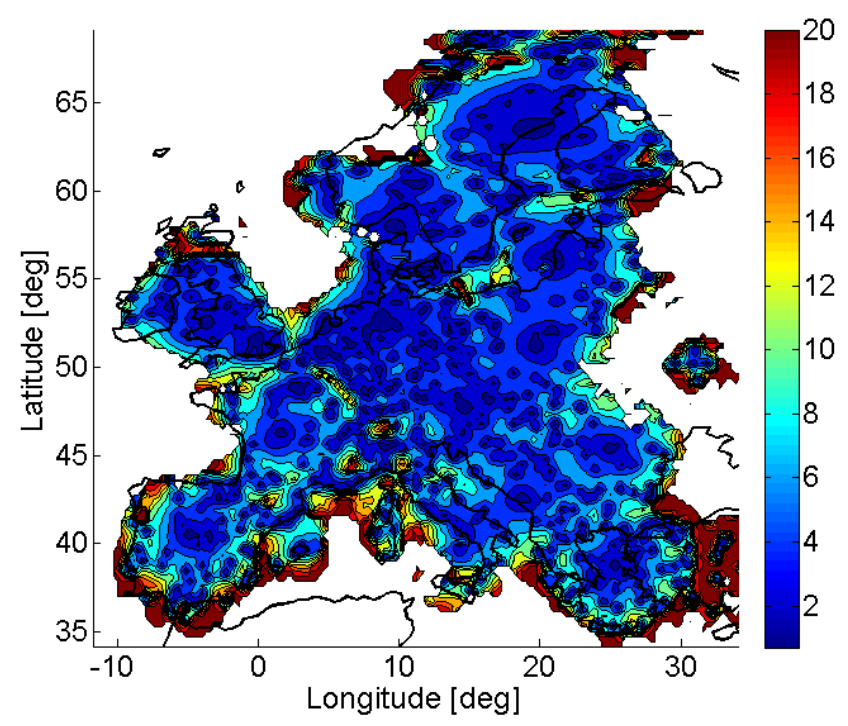

Figure 7. HDOP at 20.000 ft. with DME and HAPS

visible stations is quite limited. As seen in Figure 6 this fact leads to a large value of Horizontal Dilution Of Precision (HDOP) in many parts of Europe, and therefore we cannot achieve a relatively homogenous HDOP service area in many parts of the continent.

In this sense, the HAPS could help to enhance the stations coverage and thus, the HDOP in areas where it is more necessary. In Figure 7, we can see the HDOP that can be obtained at an altitude of 20.000 $\mathrm{ft}$. considering all the DME stations and 14 HAPS that have been spread above Europe at $20 \mathrm{~km}$ high. 


\section{HDOP over Airport Vicinity}

We consider the HDOP in the vicinity of the Charles-de-Gaule Paris airport in Figure 8 for a flying altitude of $5.000 \mathrm{ft}$. An approach to the airport that navigates with APNT would be only possible from certain trajectories (i.e., from the south). However, placing one HAPS above the north-west of the airport can increase the HDOP coverage area, allowing APNT navigation approaches from different directions. The HDOP enhancement can be seen in Figure 9.

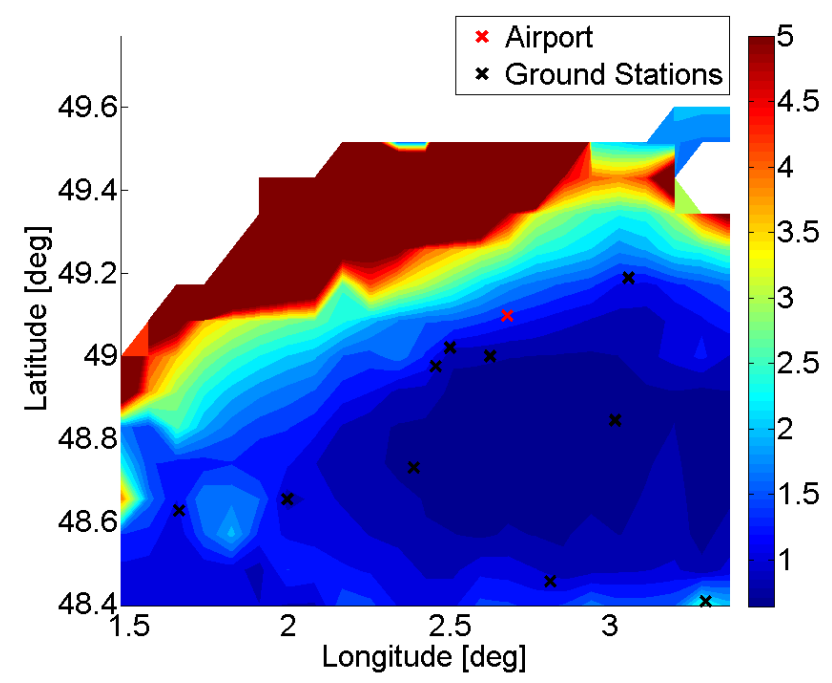

Figure 8. HDOP at 5.000 ft. with DME

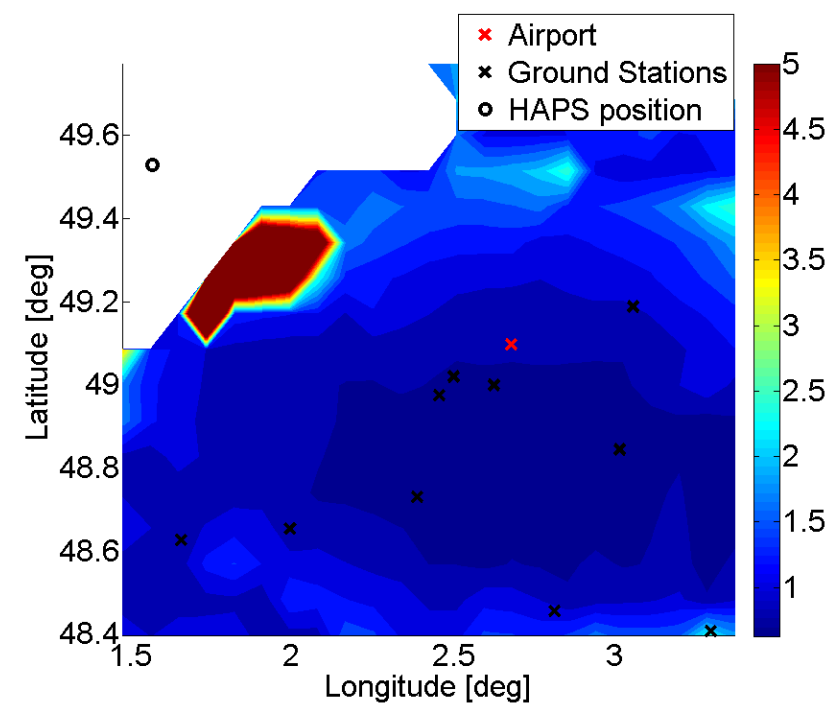

Figure 9. HDOP at $5.000 \mathrm{ft}$. with DME and HAPS

\section{Position Error Propagation}

In this section, we address the sensitivity of the user position accuracy with respect to the accuracy of the HAPS position. This first analysis will provide the guidance and control requirements of the platform. These requirements depend on different parameters like the density of the ground network and especially the geometric weight of the HAPS range with respect to the other ground ranges. We derive the instantaneous linearized error equation of the user as follows:

$$
\Delta \mathbf{y}=\mathbf{G} \Delta \mathbf{x}
$$

where $\Delta \mathbf{y}$ is the error in the pseudorange, $\Delta \mathbf{x}$ is the $4 \times 1$ vector with the position and receiver clock error and $\mathbf{G}$ is the geometric matrix. After multiplying both sides of Equation (9) by the pseudo-inverse of $\mathbf{G}$ we obtain:

$$
\Delta \mathbf{x}=\left(\mathbf{G}^{\mathrm{T}} \mathbf{G}\right)^{-1} \mathbf{G}^{\mathrm{T}} \Delta \mathbf{y} .
$$

Let's split $\mathbf{G}$ and $\Delta \mathbf{y}$ into their components relative to the HAPS (using simplified index ' $h$ ') and those relative to the visible ground ranging sources (using simplified index ' $\mathrm{g}$ '). Let's write:

$$
\Delta \mathbf{y}=\left[\begin{array}{ll}
\Delta \mathbf{y}_{\mathrm{h}}^{\mathrm{T}} & \Delta \mathbf{y}_{\mathrm{g}}^{\mathrm{T}}
\end{array}\right]^{\mathrm{T}}
$$

and

$$
\mathbf{G}=\left[\begin{array}{ll}
\mathbf{G}_{\mathrm{h}}^{\mathrm{T}} & \mathbf{G}_{\mathrm{g}}^{\mathrm{T}}
\end{array}\right]^{\mathrm{T}} .
$$

Equation (10) can be written in an implicit form by multiplying both sides with $\mathbf{G}^{\mathrm{T}}$ in order to express the equation using the above defined components:

$$
\begin{aligned}
\mathbf{G}^{\mathrm{T}} \mathbf{G} \Delta \mathbf{x} & =\mathbf{G}^{\mathrm{T}} \Delta \mathbf{y} \\
\left(\mathbf{G}_{\mathrm{h}}^{\mathrm{T}} \mathbf{G}_{\mathrm{h}}+\mathbf{G}_{\mathrm{g}}^{\mathrm{T}} \mathbf{G}_{\mathrm{g}}\right) \Delta \mathbf{x} & =\mathbf{G}_{\mathrm{h}}^{\mathrm{T}} \Delta \mathbf{y}_{\mathrm{h}}+\mathbf{G}_{\mathrm{g}}^{\mathrm{T}} \Delta \mathbf{y}_{\mathrm{g}} .
\end{aligned}
$$

Let's decompose now the error $\Delta \mathbf{y}_{\mathrm{h}}$ into an error due to the HAPS position and clock error $\Delta \mathbf{e}_{\mathrm{h}}$ and the other error sources $\Delta \mathbf{r}_{\mathrm{h}}$. In fact $\Delta \mathbf{e}_{\mathrm{h}}$ is the projection of the 4D HAPS error (3D for position and 1D for the clock) in the line of sight direction. If we call $\Delta \mathbf{x}_{\mathrm{h}}$ the 4D HAPS error vector, we have the following relation:

$$
\Delta \mathbf{e}_{\mathrm{h}}=\mathbf{G}_{\mathrm{h}} \Delta \mathbf{x}_{\mathrm{h}} .
$$

We replace the terms defined in Equation (14):

$$
\begin{aligned}
& \left(\mathbf{G}_{\mathrm{h}}^{\mathrm{T}} \mathbf{G}_{\mathrm{h}}+\mathbf{G}_{\mathrm{g}}^{\mathrm{T}} \mathbf{G}_{\mathrm{g}}\right) \Delta \mathbf{x}=\mathbf{G}_{\mathrm{h}}^{\mathrm{T}}\left(\Delta \mathbf{e}_{\mathrm{h}}+\Delta \mathbf{r}_{\mathrm{h}}\right)+\mathbf{G}_{\mathrm{g}}^{\mathrm{T}} \Delta \mathbf{y}_{\mathrm{g}}(16) \\
& \left(\mathbf{G}_{\mathrm{h}}^{\mathrm{T}} \mathbf{G}_{\mathrm{h}}+\mathbf{G}_{\mathrm{g}}^{\mathrm{T}} \mathbf{G}_{\mathrm{g}}\right) \Delta \mathbf{x}=\mathbf{G}_{\mathrm{h}}^{\mathrm{T}} \mathbf{G}_{\mathrm{h}} \Delta \mathbf{x}_{\mathrm{h}}+\mathbf{G}_{\mathrm{h}}^{\mathrm{T}} \Delta \mathbf{r}_{\mathrm{h}}+\mathbf{G}_{\mathrm{g}}^{\mathrm{T}} \Delta \mathbf{y}_{\mathrm{g}}
\end{aligned}
$$


If we multiply both sides of Equation (17) by $\mathbf{K}_{\mathrm{h}}=$ $\left(\mathbf{G}_{\mathrm{h}}^{\mathrm{T}} \mathbf{G}_{\mathrm{h}}\right)^{-1}$, we obtain the following equation:

$$
\left(\mathbf{I}+\mathbf{K}_{\mathrm{h}} \mathbf{G}_{\mathrm{g}}^{\mathrm{T}} \mathbf{G}_{\mathrm{g}}\right) \Delta \mathbf{x}=\Delta \mathbf{x}_{\mathrm{h}}+\mathbf{K}_{\mathrm{h}} \mathbf{G}_{\mathrm{h}}^{\mathrm{T}} \Delta \mathbf{r}_{\mathrm{h}}+\mathbf{K}_{\mathrm{h}} \mathbf{G}_{\mathrm{g}}^{\mathrm{T}} \Delta \mathbf{y}_{\mathrm{g}}
$$

Let's call $\mathbf{P}=\left(\mathbf{I}+\mathbf{K}_{\mathrm{h}} \mathbf{G}_{\mathrm{g}}^{\mathrm{T}} \mathbf{G}_{\mathrm{g}}\right)^{-1}$, Equation (18) is then

$$
\Delta \mathbf{x}=\mathbf{P} \Delta \mathbf{x}_{\mathrm{h}}+\mathbf{P} K_{\mathrm{h}} \mathbf{G}_{\mathrm{h}}^{\mathrm{T}} \Delta \mathbf{r}_{\mathrm{h}}+\mathbf{P} \mathbf{K}_{\mathrm{h}} \mathbf{G}_{\mathrm{h}}^{\mathrm{T}} \Delta \mathbf{y}_{\mathrm{g}}
$$

As a result, in Equation (19) we have separated in the final position error the contribution due to the HAPS position and timing error, the HAPS additional ranging errors and the errors due to the ground stations ranges. The nature of the ranging errors is similar when the source is on ground or a HAPS, that is, there are mainly due to tropospheric delays and multipath. Therefore, the new error contribution in our hybrid ground-stratospheric system comes from the use of the HAPS as a moving pseudolite. In fact, the final error will depend on the relation between three factors: the accuracy of the positioning and timing system used by the HAPS, how predictable are the dynamics of the platform and the rate at which the platform will transmit its own ephemeris. A deeper analysis of this aspects are out of the scope of this document, but we can identify from a geometrical point of view, how this position and time error of the platform will be amplified depending on the position of the ground stations, HAPS and user, that is, the sensitivity factor is finally:

$$
\frac{\partial \mathbf{x}}{\partial \mathbf{x}_{\mathrm{h}}}=\mathbf{P}=\left(\mathbf{I}+\left(\mathbf{G}_{\mathrm{h}}^{\mathrm{T}} \mathbf{G}_{\mathrm{h}}\right)^{-1} \mathbf{G}_{\mathrm{g}}^{\mathrm{T}} \mathbf{G}_{\mathrm{g}}\right)^{-1} .
$$

\section{Further Applications of HAPS for APNT}

As shown before, HAPS allow covering a large service area. This is not only true for airborne users but also ground based receivers. Especially for scenarios in which -seen from the ground based receiverthe HAPS is visible at a sufficient high elevation the propagation conditions are quite good in terms of low multipath impact. Both aspects, coverage and signal quality, make further applications of HAPS even more interesting. Two fundamental ideas of that kind will be shortly discussed in the following.

\section{Time Synchronization}

Most proposals for APNT incorporate the concept of pseudoranging between ground stations and airborne users as a basis of position estimation. This requires the sufficient synchronization of the mentioned ground stations. Since APNT systems are supposed to be fully operational even in cases of severe GNSS outages, classic GNSS based means of synchronization can no longer be assumed to be available. Other fixed wire or satellite based alternatives are also in question due to feasibility and/or costbenefit concerns [6]. HAPS which offer an APNT service can also help to provide the necessary means for synchronization. For this purpose a synchronization signal which is not in the L-band is transmitted from the HAPS to the ground. Knowing the position of the HAPS and its own position each ground station can synchronize its local time reference to the received synchronization signal. Since all ground stations in view of the HAPS can synchronize to the same synchronization signal the relative synchrony of the ground stations can be ensured. Avoiding the L-band for transmission of the synchronization signals avoids correlated signal outages of GNSS and of the synchronization backbone of the APNT system. Using HAPS both for pseudoranging and for synchronization within the APNT system is a costbeneficial solution which has to be further elaborated in a future paper. Also the application for the novel synchronization means for other applications will be discussed.

\section{Integrity Monitoring}

HAPS offer some quite interesting benefits:

1) even in cases of GNSS outage due to local jamming it can be assumed that GNSS positioning of the HAPS itself is still possible due to the large distance to the jammer and the pointing of the GNSS antenna opposite to the direction of the interferer and

2) the propagation conditions in terms of multipath between APNT ground stations and HAPS can be assumed to be quite good.

Consequently, the integrity of the APNT signals transmitted by the ground stations can be well observed and monitored by the HAPS. This offers the opportunity to monitor integrity of the APNT systems by suitable monitoring facilities onboard of 
the HAPS. This facilities should be able to at least monitor signal anomalies (deformations, biases, evil waveforms etc.) as well as synchronization errors (clock events, uncompensated drifts etc.). However, it can also monitor undesired propagation effects and can help to gain a higher statistical significance in nominal error distributions which help to drive further developments of the APNT system. Detailed concepts for HAPS based integrity monitoring for APNT will be the topic of a further paper.

\section{Discussions}

We have observed that we can cover a large area with only one HAPS and therefore they can be placed to enhance the APNT service in sparse ground stations areas. Due to the proximity to the ground (compared to a satellite), in order to satisfy the link budget, the antenna design will not be straight forward and will require a dedicated study. We have seen the improvement in terms of Horizontal Dilution Of Precision when adding one or more HAPS. In the study performed in this paper, the position and number of HAPS used has been decided trying to increase the overall HDOP service area within Europe, an specific design would be required to find the optimal positions. From the sensitivity analysis, we have seen that it is possible to derive how the HAPS position error contributes to the final user position error. This could be used to set the station keeping capabilities requirements of the platform for this application.

\section{Conclusions}

The concept presented in this paper about using High Altitude Platform Systems (HAPS) in the context of Alternative Position Navigation and Timing (APNT) opens a new application for the stratospheric platforms and propose a means for solving some APNT current issues in geometry diversity and time synchronization. The improvement shown in terms of Horizontal Dilution Of Precision (HDOP) would enhance the horizontal navigation service area expected from APNT giving more freedom to the Air Traffic Management (ATM) to organize the air traffic in case of GNSS unavailability. Finally, a very interesting application of HAPS in the context of APNT is the possible capability of the HAPS to perform integrity monitoring of the ground stations, being a new service not considered in the current APNT proposals.

\section{Acknowledgments}

We would like to thank Anja Grosch for reviewing the paper and her fruitful comments and suggestions. We would also like to thank the aeronautics program directorate of DLR for funding this research.

\section{References}

[1] SESAR, http://www.sesarju.eu.

[2] NextGen, http://www.faa.gov/nextgen.

[3] M. Narins, P. Enge, B. Peterson, S. Lo, Y. Chen, D. Akos, and M. Lombardi, "The need for a robust and precise time and frequency alternate to global navigation satellite systems," The Journal of Air Traffic Control, vol. 55, 40:46, Nov. 2012.

[4] K. Li and W. Pelgrum, "Enhanced DME carrier phase: Concepts, implementation, and flight-test results," Journal of The Institute of Navigation, vol. 60, no. 3, 2013.

[5] S. C. Lo, B. Peterson, D. Akos, M. Narins, R. Loh, and P. Enge, "Alternative Position Navigation \& Timing (APNT) Based on Existing DME and UAT Ground Signals," in Proceedings of the ION GNSS 2011, 2011.

[6] S. Lo, D. Akos, and J. Dennis, "Time source options for alternate positioning navigation and timing (APNT)," Federal Aviation Administration, Tech. Rep., Aug. 2012.

[7] D. Shutin, N. Schneckenburger, and M. Schnell, "LDACS1 for APNT - planning and realization of a flight measurement campaign," in 31st Digital Avionics Systems Conference (DASC 2012), Williamsburg, USA, Oct. 2012.

[8] S. Lo, "Pseudolite Alternatives for Alternate Positioning, Navigation, and Timing (APNT)," Federal Aviation Administration (FAA), Whitepaper, Aug. 2012.

[9] A. Aragón-Zavala, J. L. Cuevas-Ruíz, and J. A. Delgado-Penín, High-Altitude Platforms for Wireless Communications. John Wiley \& Sons, 2008.

[10] S. Karapantazis and F.-N. Pavlidou, "Broadband Communications via High-Altitude Platforms: A Survey," IEEE Communications Surveys and Tutorials, vol. 7, First Quarter 2005.

[11] V. Welch, G. Baird, D. P. Cadogan, and S. E. Scarborough, "A Novel Concept for Stratospheric Communications and Surveillance: Star-Light," in AIAA Balloon Systems Conference, 2007. 
[12] I. Ozimek, T. Javornik, and F. Dovis, "Navigation-Related Services over Stratospheric Platform," Electrotechnical Review, Ljubljana, Slovenija, vol. 71(3), pp. 96-102, 2004.

[13] J. Wang, "Pseudolite applications in positioning and navigation: Progress and problems," Journal of Global Positioning Systems, vol. 1, pp. 48-56, 2002. [14] F. Dovis, L. Lo Presti, and P. Mulassano, "Support infrastructures based on high altitude platforms for navigation satellite systems," IEEE Wireless Communications, Oct. 2005.

[15] M. Leonardi, S. Spinelli, and G. Galati, "ADSB/MLAT surveillance system from High Altitude Platform Systems," in Proceedings of ESAV'11, 2011, pp. $153-158$.

[16] C. A. Balanis, Antenna Theory - Analysis and Design. John Wiley \& Sons, Inc., 2005.

[17] G. Minatti, S. Maci, P. De Vita, A. Freni, and M. Sabbadini, "A circularly-polarized isoflux antenna based on anisotropic metasurface," Antennas and Propagation, IEEE Transactions on, vol. 60, no. 11, pp. 4998-5009, Nov. 2012, ISSN: 0018-926X. DOI: 10.1109/TAP.2012.2208614.

[18] J. del Rio, A. Nubla, L. Bustamante, F. Vila, K. Klooster, and A. Frandsen, "Novel isoflux antenna alternative for leo satellites downlink," in Microwave Conference, 1999. 29th European, vol. 3, Oct. 1999, pp. 154-157. DOI: 10.1109/EUMA.1999.338551.

[19] A. Roederer, "Antennas for space: Some recent european developments and trends," in Applied Electromagnetics and Communications, 2005. ICECom 2005. 18th International Conference on, 2005, pp. 18. DOI: 10.1109/ICECOM.2005.204908.

\section{Email Addresses}

Contact email: Omar.GarciaCrespillo@dlr.de Other emails: \{firstname.surname\} @dlr.de

34th Digital Avionics Systems Conference September 13-17, 2015 